\title{
Tumor neuroendocrino, mucocele y adenoma tubulo-velloso: tres lesiones infrecuentes en el apéndice cecal
}

\author{
Neuroendocrine tumours, mucocele, and tubulovillous adenoma: three uncommon \\ lesions in the cecal appendix
}

\begin{abstract}
Alberto Piamo Morales', Digna Chávez Jiménez', Isnerio Arzuaga Anderson', Daisy Ferrer Marrero², Lourdes Palma Machado', Sotolongo Montano Loandy ${ }^{1}$
\end{abstract}

\begin{abstract}
Resumen
Introducción: los tumores del apéndice cecal constituyen un grupo heterogéneo de neoplasias con evolución y pronóstico variables; representan una pequeña parte de todas las neoplasias gastrointestinales y de las apendicetomías. Objetivo: realizar la comunicación de la presentación de tumores infrecuentes del apéndice cecal y revisar la literatura con énfasis la importancia del diagnóstico histopatológico. Presentación de caso: durante la necropsia del paciente HC: 199584 se realizó el diagnóstico histopatológico de un tumor neuroendocrino y mucocele apendicular y en la pieza quirúrgica del paciente HC: 223360 correspondiente a una apendicectomía se realizó el diagnóstico de adenoma tubulo-velloso, dadas que estas lesiones son poco frecuente se decide su comunicación y la revisión del tema en la literatura en idioma inglés y español a través de las bases de datos MEDLINE/PubMed, Elsevier, SciELO, EBSCO, Hinari y búsqueda avanzada en Google Scholar, encontrándose un total de 29 publicaciones entre 1990 a 2019. Conclusiones: el diagnóstico de tumores del apéndice cecal se realiza en forma incidental en el estudio anatomopatológico después de una apendicectomía por apendicitis aguda, es por ello que siempre se debe realizar el estudio histológico de la pieza quirúrgica, no solo para confirmar o negar el diagnostico que motivo la apendicetomía, sino para descartar la presencia de lesiones neoplásicas benignas o malignas.
\end{abstract}

Palabras clave: apendicitis aguda; tumor neuroendocrino; mucocele; adenoma tubulo-velloso apendicular.

\begin{abstract}
Introduction: Tumors of the cecal appendix constitute a heterogeneous group of neoplasms with variable evolution and prognosis; they represent a small part of all gastrointestinal neoplasms and appendectomies. Objective: To communicate the presentation of infrequent tumours of the cecal appendix and to review the literature with emphasis on the importance of histopathological diagnosis. Case presentation: During the necropsy of patient HC: 199584, a histopathological diagnosis of carcinoid tumours and appendicular mucocele was made, and in the surgical specimen of patient HC: 223360 correspondings to an appendectomy, the diagnosis of tubulovillous adenoma were made, given that These lesions are rare, it is decided to communicate and review the subject in the literature in English and Spanish through the MEDLINE / PubMed, Elsevier, SciELO, EBSCO, Hinari and Advanced Search in Google Scholar databases, finding a total of 29 publications between 1990 to 2019. Conclusions: The diagnosis of tumours of the cecal appendix is made incidentally in the pathological study after an appendectomy for acute appendicitis, which is why the histological study of the surgical piece should always be carried out, not only to confirm or deny the diagnosis. What reason for the appendectomy but to rule out the presence of benign or malignant neoplastic lesions.
\end{abstract}

Keywords: acute appendicitis; carcinoid tumour; mucocele; appendicular tubulovillous adenoma.

Fecha de envío: 2021-01-26 - Fecha de aceptación: 2021-08-24

(1) Departamento de Anatomía Patológica. Hospital Clínico Quirúrgico Docente "Joaquín Albarrán", La Habana-Cuba.

(2) Departamento de Medios Diagnósticos. Facultad de CM "ICBP Victoria de Girón". Universidad de Ciencias Médicas. La Habana. Cuba.

Autor de correspondencia: b51amazonas@gmail.com 
Piamo et al.

\section{Introducción}

Los tumores apendiculares son una patología rara; la mayoría de las veces, un hallazgo transoperatorio. Estas neoplasias se encuentran en el $1 \%$ de los especímenes de apendicectomía y representan sólo alrededor del $0,5 \%$ de los cánceres intestinales, los principales tipos histológicos son los tumores neuroendocrinos, adenocarcinomas, adenocarcinoides, cistoadenomas y cistoadenocarcinomas (Buchholtz et al., 2010).

Las neoplasias epiteliales apendiculares se encuentran en 0,5\% de todas las neoplasias gastrointestinales y en $1 \%$ de las apendicectomías (Sugarbaker, 2006). Una de éstas son los adenomas, los cuales producen escasos o ningún síntoma; aunque algunos son obvios en la cirugía, la mayoría se diagnostica únicamente en el estudio histopatológico (Murphy et al., 2006).

El tumor neuroendocrino representa el 20\% de las neoplasias apendiculares, suponiendo esta localización el 16\% de los tumores neuroendocrinos gastrointestinales, presentándose entre el 0,02 y el 1,5\% de todas las cirugías apendiculares (Guelmes et al., 2017).

El mucocele del apéndice es casi siempre un hallazgo, por lo que los pacientes son operados generalmente por otras causas, como ocurrió en nuestros casos, por lo que ha sido considerado también como una lesión incidental. Es por ello que se realiza la comunicación de la presentación de tres tumores infrecuentes del apéndice cecal con una revisión de la literatura, con énfasis la importancia del diagnóstico histopatológico.

\section{Materiales y métodos}

Como contribución a la escasa frecuencia de tumores del apéndice cecal reportados en la literatura se presentan dos casos recientemente diagnosticados en el Hospital Docente Clínico Quirúrgico “Joaquín Albarrán" de La Habana, en los cuales se hallaron un tumor neuroendocrino, un mucocele (caso 1) y un adenoma tubulo-velloso (caso 2). Se realiza una búsqueda en idioma inglés y español a través de las bases de datos MEDLINE/PubMed, Elsevier, SciELO, EBSCO, Hinari y búsqueda avanzada en Google Scholar, encontrándose un total de 29 publicaciones en el período comprendido entre los años 1990 al 2019.

\section{Presentación de caso}

Se presenta dos casos en los cuales, al concluir el estudio histopatológico del apéndice cecal se demostró la presencia de tres lesiones poco frecuentes localizadas en este órgano; el tumor neuroendocrino y el mucocele, ambas pertenecen al caso $N^{\circ} 1$ y el adenoma tubulo-velloso, en el caso № 2 .

\section{Caso № 1: tumor neuroendocrino y mucocele}

Paciente con No de historia clínica: 199584, masculino de 88 años de edad, con antecedentes de cardiopatía isquémica crónica y diabetes mellitus tipo 2, que es ingresado por presentar cuadro de angina de pecho inestable y tras una estadía hospitalaria de 3 días en el Servicio de Cuidados Coronarios, fallece. Luego de la autorización de los familiares se procede a la realización de la autopsia clínica, la cual reveló, como causa de muerte, la presencia de un infarto agudo de miocardio de 72 horas de evolución en cara posterolateral extenso; además, llamó la atención la presencia de la dilatación del apéndice cecal, cuyas características macroscópicas se pueden observar en la figura $1 \mathrm{~A}$, en donde se aprecia el apéndice cecal de $8 \times 3 \mathrm{~cm}$ con una superficie serosa lisa, brillante sin otras alteraciones, que a nivel de la grasa mesocólica no se observa ganglios linfáticos; en su porción media se encuentra dilatada, al corte a ese nivel (Figura 1-B), se observa un acúmulo de material mucoide traslucido de consistencia gelatinosa.

En el estudio histopatológico del apéndice cecal, se describió presencia de luz ocupada por material basófilo homogéneo que alterna con áreas de aspecto laminar (Figura 2-A) en cuyo espesor se observan múltiples acúmulos puntiformes intensamente basófilos que se corresponden con calcificación (Figura 2-B); pérdida del epitelio mucoso y pared muscular con presencia de ligero infiltrado inflamatorio linfocitario.

En el corte realizado a nivel del extremo distal (punta) se observan acúmulos de células rodeados por un estroma fibroso con un aspecto glomeruloide (Figura 3-A); las células presentan características uniformes en tamaño y forma, con escaso citoplasma y núcleos con cromatina laxa granular algunos con aspecto en sal y pimienta (Figura 3-B); no se encontró actividad mitótica, ni necrosis tumoral. El estudio inmunohistoquímico reveló una tinción citoplasmática fuertemente positiva para cromogranina de todas las células tumorales, las cuales se pueden apreciar en la luz del órgano e infiltrando la pared muscular extendiéndose algunos grupos de células hasta la serosa (Figura 4).

Como conclusión diagnóstica de la lesión encontrada en la necropsia, se informó que correspondía a mucocele simple (quiste de retención) y tumor neuroendocrino de apéndice cecal infiltrante hasta la serosa. 
Piamo et al.

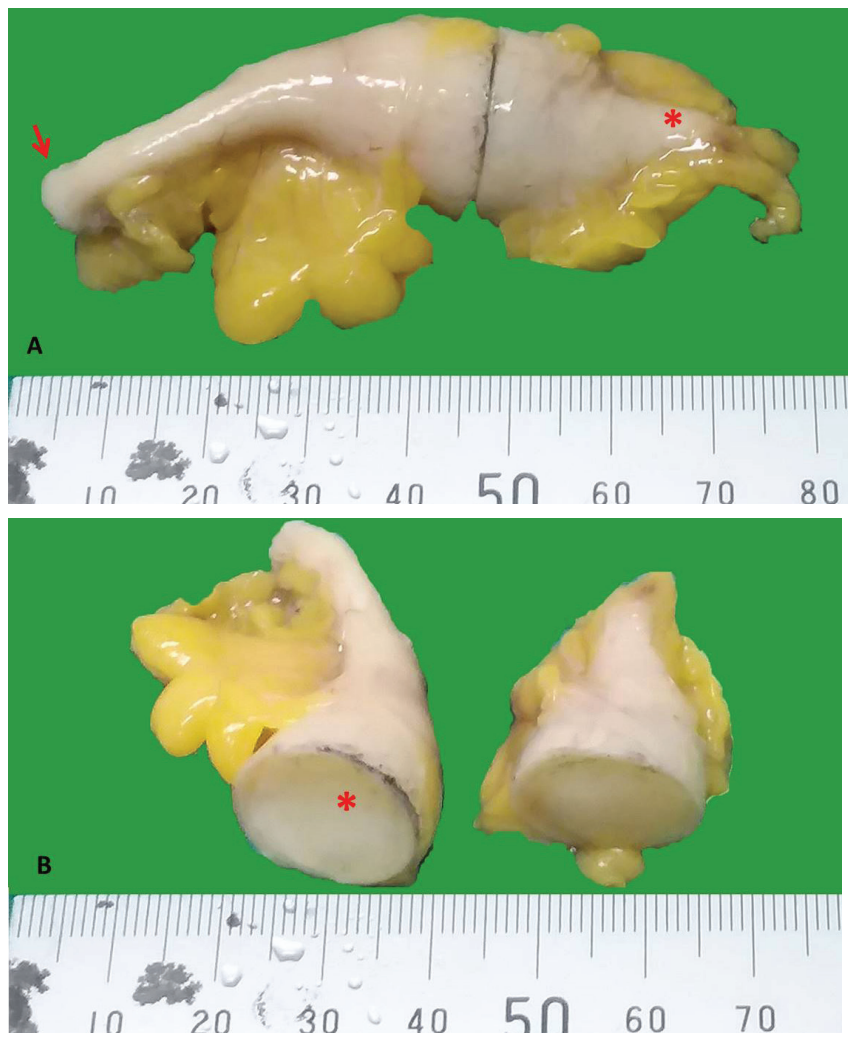

Figura 1: Aspecto macroscópico de apéndice cecal. A: Dilatación de su porción media (flecha: extremo proximal; asterisco extremo distal). B: Corte transversal a nivel de porción media con material mucoide en la luz (asterisco).
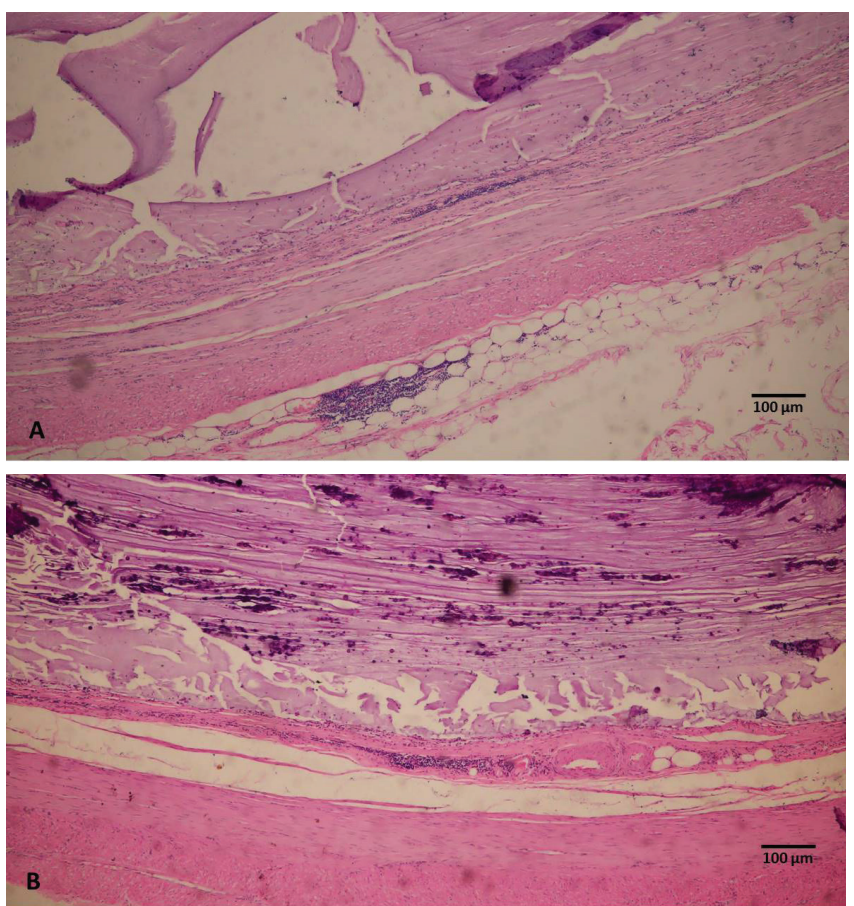

Figura 2: Pared muscular y luz de apéndice cecal. A: Pérdida de la mucosa y abundante material basófilo (mucina) en la luz (10X). B: múltiples calcificaciones pequeñas en el espesor de la mucina (10X).
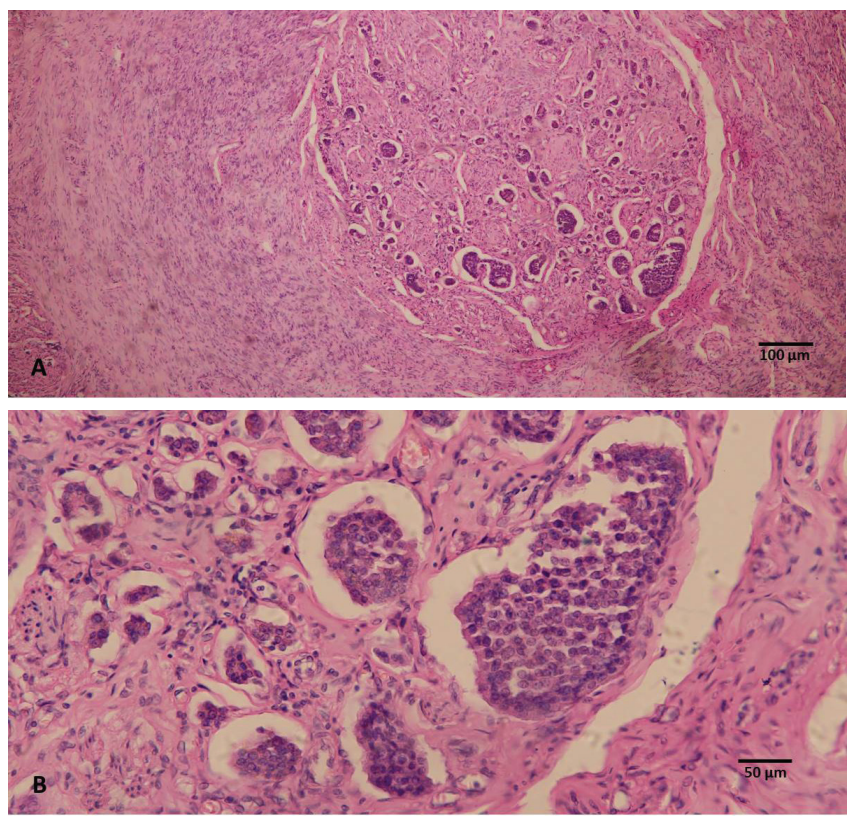

Figura 3: Tumor neuroendocrino de apéndice cecal. A: Acúmulos de células rodeados por un estroma fibroso (10X). B: Células de forma y tamaño uniformes con escaso citoplasma y núcleos con cromatina laxa granular con aspecto en sal y pimienta (15X).

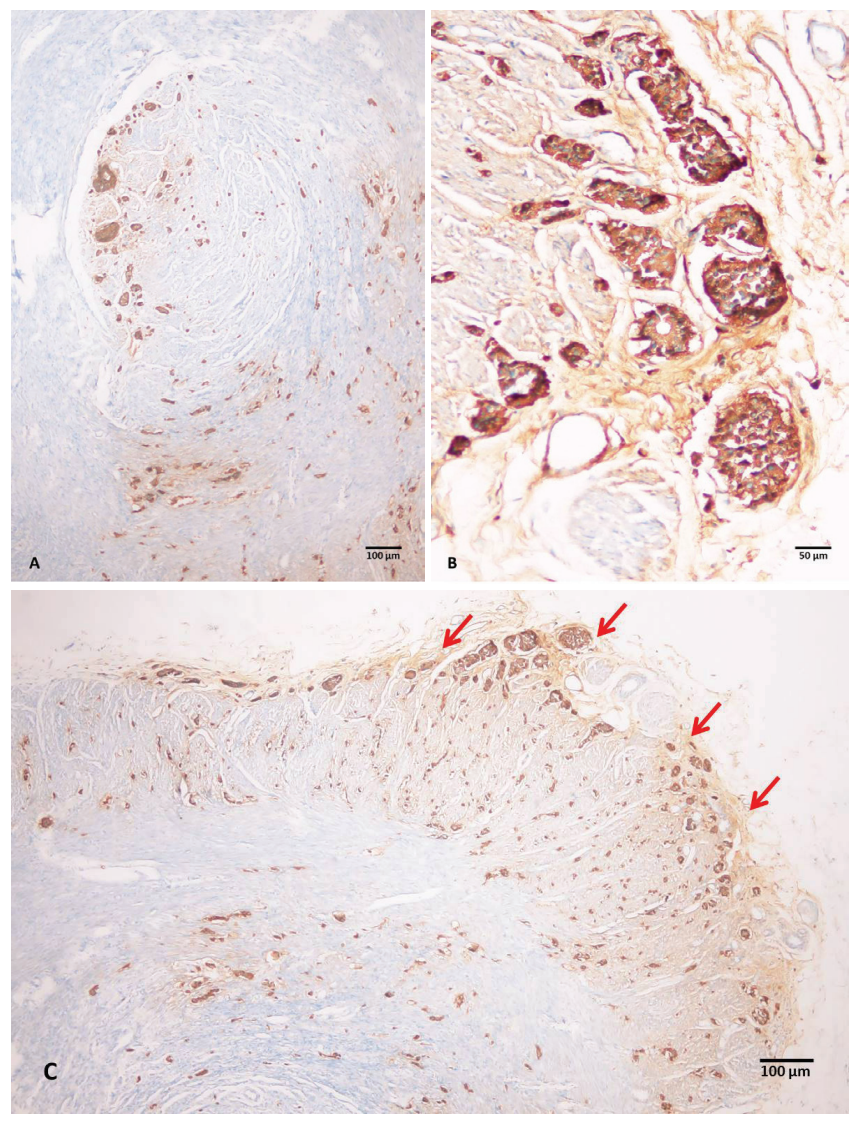

Figura 4: Tinción de inmunohistoquímica con cromogranina. A: Células tumorales en la luz del apéndice (10X). B: Grupos de células tumorales intensamente positivas en la pared muscular (15X). C: Presencia de células malignas que infiltran hasta la serosa peritoneal (10X). 
Piamo et al.

\section{Caso No 2: Adenoma tubulo-velloso}

Paciente con Nº de historia clínica: 223360, femenina de 69 años de edad, con antecedentes de diabetes mellitus tipo 2, que 24 horas previas a su ingreso presentó dolor abdominal en epigastrio que posteriormente se trasladó hacia fosa iliaca derecha con aumento progresivo de intensidad asociado a vómitos, anorexia y fiebre; al examen físico, presentaba dolor a la palpación profunda en fosa iliaca derecha con signo de blumberg positivo, así como exámenes complementarios en los que se pudo constatar una leucocitosis a predominio de neutrófilos. Se diagnosticó una apendicitis aguda, por lo cual se realizó apendicetomía abierta y es dada de alta sin complicaciones al segundo día del postoperatorio.

En el estudio anatomopatológico de la pieza quirúrgica, se describió, desde el punto de vista macroscópico un apéndice cecal de $10 \times 1 \mathrm{~cm}$ con superficie serosa brillante con moderada congestión vascular, no se observan depósitos de fibrina ni soluciones de continuidad; a los múltiples cortes se aprecia pared de $0,4 \mathrm{~cm}$ de grosor y luz de $0,6 \mathrm{~cm}$ de diámetro con material fecaloideo pastoso.

Al examen microscópico se observó presencia de infiltrado inflamatorio a predominio de polimorfonucleares neutrófilos entre los haces musculares de la pared extendiéndose hasta la serosa y la grasa periapendicular (Figura 5-A), además de folículos linfoides de tamaño variable en la submucosa $y$, a nivel de la mucosa se aprecia presencia de glándulas aumentadas en número algunas de las cuales con extensiones similares a vellosidades con un epitelio que muestra displasia de bajo grado (Figura 5-B).

El diagnóstico de biopsia confirmó el diagnóstico de apendicitis aguda con periapendicitis así como la existencia de adenoma tubulo-velloso con displasia de bajo grado localizado hacia el tercio distal del apéndice. No signos de malignidad.

\section{Discusión}

Dentro de la patología apendicular predominan los procesos inflamatorios (Baltazar et al., 2019); y los tumores constituyen un pequeño grupo, con una baja incidencia, la que varía aproximadamente entre el 0,2 y 0,5\% de todas las neoplasias gastrointestinales, y se manifiestan con una frecuencia menor al $2 \%$ de todos los pacientes sometidos a apendicectomía de urgencia por apendicitis aguda (Martínez et al., 2018). En el estudio de Butte et al. (2007) se observó que los tumores apendiculares son poco frecuentes, ya que se diagnosticaron en el $0,8 \%$ de los enfermos sometidos a una apendicectomía. La edad media de presentación de estos tumores se sitúa en la sexta década de la vida y son más frecuentes en varones (Rouchad et al., 2014).
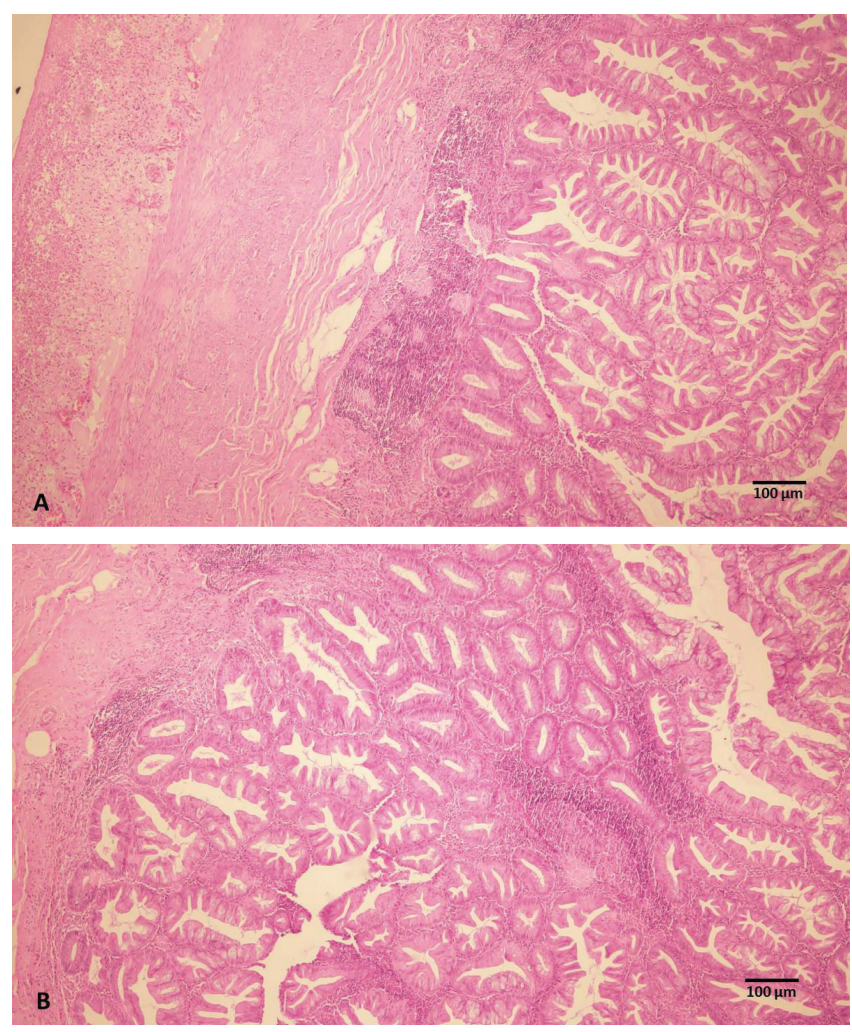

Figura 5: Apendicitis aguda con periapendicitis. A: Presencia de infiltrado inflamatorio de neutrófilos en la pared muscular y en la serosa (10X). B: Aumento del número de glándulas algunas con displasia (10X).

A pesar de ello, su importancia radica en que excepcionalmente son diagnosticados antes o durante la cirugía, y en los casos en que se sospecha el diagnóstico intraoperatorio pueden surgir dudas respecto al tratamiento quirúrgico de elección (Esmer et al., 2004). En ocasiones, tales tumores son identificados durante la necropsia como en el primer caso descrito.

Pueden existir tumores benignos y malignos (Esmer et al., 2004). La presentación más común de neoplasias malignas de apéndice es el tumor neuroendocrino, con un promedio de un $66 \%$. En el segundo lugar se tiene el cistadenocarcinoma con un $20 \%$ y en tercer lugar los adenocarcinomas con un 10\%. Las masas apendiculares benignas también se han reportado de forma rara y dentro de los subtipos histológicos se tienen neoplasias epiteliales mucinosas, adenomas, cistadenomas y mucocele neoplásico benigno (Madrigal et al., 2018).

El tumor neuroendocrino de apéndice cecal representa el 16\% con respecto a los que se localizan en otros órganos del intestino medio (Haliberto et al., 2014). En un estudio de más de 34000 apendicectomías la prevalencia de los tumores neuroendocrinos fue de 0,32\% (Rodríguez et al., 2015). En el Hospital Docente 
Clínico Quirúrgico "Joaquín Albarrán" durante el año (2018) en que se hizo el diagnóstico de tumor neuroendocrino de apéndice que se presenta, se realizaron un total de 242 apendicectomías, para una frecuencia en dicho centro de salud para los tumores neuroendocrinos de $0,4 \%$.

Generalmente están localizados en la punta del apéndice (62-78\%) y tienen un diámetro menor de un centímetro en el $70-95 \%$, lo que también coincide con el caso aquí presentado. Se pueden hallar también de forma incidental después de otros procederes como colectomía, colecistectomía y salpingectomía, y en estudios post-morten en 1\% (Ganim et al., 2012).

En 1910 Hubschmann observó que las células de estos tumores eran idénticas a las células enterocromafines descritas en 1870 por Kultschitzky en las criptas de Lieberkhun. En 1914, Gosset y Masson utilizaron técnicas de impregnación de plata y demostraron que los tumores neuroendocrinos pueden surgir a partir de las células enterocromafines dentro de las glándulas de Lieberkühn y comprobaron que sus gránulos reaccionan con sales de plata y así, establecieron la caracterización de los tumores neuroendocrinos como neoplasia de células argentafines (Haliberto et al., 2014).

Esta neoplasia se detecta con mayor frecuencia en los pacientes alrededor de los cuarenta años con una incidencia leve pero consistentemente mayor en las mujeres (Wagner et al., 2013).

Los tumores neuroendocrinos del apéndice, al ser raros, son difíciles de diagnosticar preoperatoriamente; es extremadamente importante el seguimiento de los pacientes con apendicitis aguda y las conclusiones del examen anatomopatológico. Igualmente, es necesario incorporar las nuevas técnicas de imágenes diagnósticas, dado que esto puede representar la toma de decisiones sobre procedimientos quirúrgicos óptimos para cada uno de los casos (Rouchaud et al., 2014). Tal es la importancia de la anterior afirmación, que en el caso № 2, se trata de una lesión proliferativa con presencia de displasia, es decir, una lesión con potencialidades de progresión hacia la malignidad.

La mayoría de ellos son pequeños, en el momento del diagnóstico; particularmente en el apéndice cecal, donde constituyen un hallazgo. Las características clínicas de los tumores neuroendocrinos gastrointestinales (Gl) varían de acuerdo con la ubicación anatómica y tipo celular (Ganim et al., 2012).

La sospecha de esta entidad es muy difícil, lo que conlleva al diagnóstico tardío y a un peor pronóstico (Hanuarys et al., 2017), ello debido a su tendencia a producir metástasis. En el estudio de Panomreongsak et al. (2000), se descubrieron metástasis tumorales en hígado, pulmón derecho, ganglios linfáticos mediastínicos y supraclaviculares derechos, además describieron por medio de microscopia electrónica gránulos neurosecretores pleomórficos en intestino medio.

Sobre la invasión a ganglios linfáticos Daskalakis et al. (2020) demostraron en un metanálisis que el tamaño del tumor mayor a 20 $\mathrm{mm}$, así como mayor a $10 \mathrm{~mm}$ y/o las invasiones vasculares, de los vasos linfáticos y perineurales se asocian con un mayor riesgo de metástasis de los ganglios linfáticos en pacientes adultos con neoplasias neuroendocrinas apendiculares. En el estudio de Brighi et al. (2020) se analizaron 435 pacientes con tumor neuroendocrino, 21 casos tenían afectación ganglionar. Similares hallazgos describieron Kleiman et al. (2015) reportando una incidencia global de enfermedad metastásica del 10\%, siendo más frecuente en hombres (75\%).

En el presente estudio no se observó afectación de la grasa mesocólica, sin embargo, RaultPetit et al. (2019) describieron de un total de 403 pacientes una afectación del mesoapéndice ( $3 \mathrm{~mm}$ ) en $5 \%$ de los casos; invasión linfovascular en $15 \%$ o perineural en $24 \%$.

Ante esta evidencia, el mayor grado tumoral y la invasión linfovascular deben considerarse como los pronosticadores de riesgo más importantes (Galanopoulos et al., 2019).

Otro tipo de neoplasias son los adenomas, los cuales se dividen en difusos, circunferenciales y localizados, (tipo nodular). El primero tiende a producir quistes enormes, de paredes delgadas y llenos de moco, por lo que se denomina cistoadenoma o mucocele mucinoso; éstos últimos representan alrededor del $8 \%$ de las neoplasias apendiculares (Nutu et al., 2017). Castañeda et al. (2009) reportaron una incidencia de tumores benignos de 0,79\% para los adenomas, 1,10\% para los mucoceles y 1,57\% para los cistoadenomas mucinosos.

El término mucocele apendicular, fue descrito por Rokitansky en 1842 y define una dilatación del apéndice vermiforme, la cual es producida por acumulación intraluminal de moco. Se trata de una entidad clínica que puede ser ocasionada por cuatro procesos patológicos subyacentes diferentes: 1. Obturación de la comunicación cecoapendicular por un fecalito o retracción cicatricial. 2. Hiperplasia mucosa focal o difusa, sin a tipias celulares. 3. Cistoadenoma mucinoso, con cierto grado de atipia celular. 4. Cistoadenocarcinoma mucinoso (Godínez et al., 2018).

El mucocele del apéndice también puede resultar de la retención fecal o pólipos del ciego que pueden obstruir el ostium del apéndice. Las causas poco frecuentes encontradas en la literatura son la endometriosis y el melanoma metastásico (Carr et al., 2016). 
Morfológicamente los mucoceles apendiculares o las neoplasias mucinosas son lesiones caracterizadas por un apéndice distendido y lleno de moco (Kehagias et al., 2016). En este se produce dilatación apendicular por mucus espeso, lo cual ocurre generalmente como resultado de obstrucción de la luz por un fecalito u otra lesión como una estenosis inflamatoria. La distensión llega a producir atrofia de las células mucosas que secretan mucus y la producción de secreciones se detiene (Crawford, 1999). Tales características son bien apreciables en el estudio histopatológico del caso № 1 que se muestra en la Figura 2.

En raras ocasiones ocurre la ruptura de un mucocele, lo que provoca derrame de contenido mucoso inocuo dentro de la cavidad peritoneal (Crawford, 1999).

Los mucoceles de retención suelen ser de pequeño tamaño, mientras los cistoadenomas y cistoadenocarcinomas pueden alcanzar un enorme desarrollo (Echenique et al., 2007). El diagnóstico suele realizarse durante la cirugía o de forma incidental en el análisis de las piezas histológicas, lo que representa menos del $1 \%$ de todas las piezas de apendicectomías (Shaib et al., 2015). Sólo el 50\% de los pacientes presentan síntomas, el resto se diagnostica como hallazgo de estudios o cirugía de otro padecimiento. En pacientes sintomáticos, el dolor abdominal localizado en fosa iliaca derecha es lo más común, seguidos de una masa abdominal, pérdida ponderal, náuseas, vómitos y apendicitis aguda, síntomas urinarios, sepsis. El pseudomixoma peritoneal presenta síntomas no específicos, y lo usual es el incremento del perímetro abdominal (Alarcón et al., 2011).

Según la clasificación de Pai \& Longacre (2005), los TMA se dividen en cistoadenoma mucinoso (CM), neoplasia mucinosa de potencial incierto maligno (NPIM), neoplasia mucinosa de bajo potencial maligno (NBPM) y adenocarcinoma mucinoso (AM). La ascitis mucinosa conocida como pseudomixoma peritoneal (PP) está presente en más del $50 \%$ de estos pacientes y su presencia indica un estadio más avanzado y un peor pronóstico. Puede presentarse como de bajo grado (adenomucinosis peritoneal difusa), o de alto grado (carcinomatosis peritoneal difusa). Actualmente, la tendencia es diferenciarlos según su grado histológico (alto o bajo), según la clasificación de la American Joint Committee on Cancer (Kaitlyn et al., 2015) queda obsoleto el término mucocele.

Al igual que la mayoría de los tumores del apéndice (benigno y maligno), el adenoma encontrado en el presente estudio, se localizaba en el tercio distal, donde es más frecuente (Butte et al., 2007).

Histológicamente son de tipo tubular usualmente y de bajo grado de displasia y cuando la tienen aparece en la base de la cripta. Pueden ser cistadenomamucinosos y con borde en sierra como los pólipos hiperplásicos (Guang et al., 2011). Es de vital importancia el examen meticuloso de la pieza (Karmarkar et al., 2008).
Su importancia clínica consiste en el compromiso vascular y parietal que desencadena la estasis venosa que producen en la luz apendicular con episodios de apendicitis aguda y que constituye una de las formas más frecuentes de urgencias quirúrgicas dentro del abdomen agudo con connotación especial en el adulto mayor, especialmente en la ancianidad, debido a que las urgencias médicas en estas edades se presentan habitualmente por enfermedades vasculares o malignas (Swank et al., 2010).

El adenoma velloso de apéndice es una entidad rara cuya forma de presentación clínica más habitual es la apendicitis aguda. La apendicectomía es el tratamiento de elección siempre que no exista degeneración maligna o invasión del borde de resección quirúrgica. Su diagnóstico requiere un seguimiento de forma periódica mediante colonoscopias (Flórez et al., 2014). En algunos casos excepcionales, el diagnóstico de un tumor apendicular se sospecha durante la cirugía o en el período preoperatorio (Beltrán et al., 2013).

\section{Conclusiones}

Los tumores apendiculares suelen presentarse como hallazgo incidental durante una intervención quirúrgica, una exploración radiológica o en el estudio anatomopatológico de una pieza de apendicectomía y su estudio histológico no solo es útil para confirmar o negar el diagnóstico que motivó la apendicetomía, sino para descartar la presencia de lesiones neoplásicas benignas o malignas.

\section{Referencias}

Alarcón G, Shuchleib A, Ylgovsky L, Padilla A, Chousleb A. \& Shuchleib S. (2011). Mucocele apendicular. Reporte de dos casos clínicos. An Med (Mex) 56, 210-7.

Baltazar I, Iñiguez C, Vázquez E, Martínez F, Ulloa J, Amezcua J, Mercado JL, Alvarado RA, Guzmán LA, López PL, Schmidt RA, Tapia CD. \& Velarde RVJ. (2019). Prevalencia de neoplasias apendiculares: Revisión clínico-patológica de apendicectomías durante 6 años. Rev Med Md. 10, 119-24.

Beltrán SM, Tapia LR, Madariaga GJ, Díaz JR, Larraín TC. \& Jaramillo RL. (2013). Tumores malignos del apéndice cecal en pacientes operados por apendicitis en la IV Región de Chile. Rev Chil Cir. 65, 509-14.

Brighi N, La Rosa S, Rossi G, Grillo F, Pusceddu S, Rinzivillo M, Spada F, Tafuto S, Massironi S, Faggiano A, Antonuzzo L, Santini D, Sessa F, Maragliano R, Gelsomino F, Albertelli M, Vernieri C, Panzuto F, Fazio N, De Divitiis C, Lamberti G, Colao A, Fave GD. \& Campana D. (2020). Morphological Factors Related to Nodal Metastases in Neuroendocrine Tumors of the Appendix: A Multicentric Retrospective Study. Ann Surg. 271, 527-33. 
Buchholtz M, Zúñiga JM, Valdés F. \& Fernández R. (2010). Carcinoide gastrointestinal. Experiencia de siete años en el Instituto Nacional del Cáncer (2000-2006). Rev Chil Cir. 62, 480-5.

Butte BJ, García DM, Torres MJ, Salinas FM, Duarte GI, Pinedo MG, Zúñiga AD. \& Llanos OL. (2007). Tumores del apéndice cecal. Análisis anatomoclínico y evaluación de la sobrevida alejada. Rev. Chilena de Cirugía 59.

Carr NJ, Cecil TD, Mohamed F, Sobin LH, Sugarbaker PH, González-Moreno S, Taflampas P, Chapman S. \& Moran BJ. (2016). Peritoneal Surface Oncology Group International. A Consensus for Classification and Pathologic Reporting of Pseudomyxoma Peritonei and Associated Appendiceal Neoplasia: The Results of the Peritoneal Surface Oncology Group International (PSOGI) Modified Delphi Process. Am J Surg Pathol. 40, 14-26.

Castañeda L, Valanci S. \& Belmonte C. (2009). Incidencia de tumores del apéndice en el Centro Médico ABC. AnMed (Mex) 54, 201-5.

Crawford JM. (1999). The Gastrointestinal tract. Cotran: Robbins Pathologic Basis of Disease 6. ed. St. Louis: WB saunders.

Daskalakis K, Alexandraki K, Kassi E, Tsoli M, Angelousi A, Ragkousi A. \& Kaltsas G. (2020). The risk of lymph node metastases and their impact on survival in patients with appendiceal neuroendocrine neoplasms: a systematic review and meta-analysis of adult and paediatric patients. Endocrine. 67, 2034.

Echenique M, Liron de Roblesa C, Amondarain J. \& Aribe X. (2007). Mucoceles apendiculares. Rev Cirugía Española 82, 251-318.

Esmer D, Martínez J, Román P, Sánchez P. \& Medina E. (2004). Tumores apendiculares. Revisión clínico-patológica de 5307 apendicectomías. Cir Ciruj 72, 375-8.

Flórez GM, Cervantes JN, Muñoz LV, Estela VL, Sánchez del Pueblo C, García AM, Talavera EP. \& Torres GA. (2014). P-392 - adenoma velloso de apéndice cecal. Cir Esp. 92, 765.

Galanopoulos M, McFadyen R, Drami I, Naik R, Evans N, Luong TV, Watkins J, Caplin M. \& Toumpanakis C. (2019). Challenging the Current Risk Factors of Appendiceal Neuroendocrine Neoplasms: Can They Accurately Predict Local Lymph Nodal Invasion? Results from a Large Case Series. Neuroendocrinology 109, 179-86.

Ganim RB. \& Norton JA. (2012). Recent advances in carcinoid pathogenesis, diagnosis and management. Gastrointest Oncol. 9, 1739.
Godínez A, Gracida N, Aguirre V, Reyes V, Martínez A, Pérez S, Villanueva HJ. \& Jiménez BB. (2018). Mucocele apendicular. Rev Hosp Jua Mex 85, 234-7.

Guang Y, Ming H, Lin Y, Chiao Y. \& Hui C. (2011). Perforated acute apendicitis resulting from appendice alvillous adenoma presenting with small bowel obstruction: a case report. Gastroenterology. 11.

Guelmes A, Sánchez C. \& Rivero N. (2017). Tumor carcinoide del apéndice cecal. Presentación de caso. Gac Méd Espirit 19.

Haliberto AB. (2014). Presentación de una paciente con tumor carcinoide del apéndice cecal. CCM 18.

Hanuarys E, Lumey N. \& Rodríguez C. (2017). Tumor carcinoide del apéndice cecal. Presentación de un caso. Medisur 15.

Kaitlyn J. (2015). Management of appendix cancer. Clin Colon Rectal Surg 28, 247-55.

Karmarkar P, Joshi A, Wilkinson A, Mahore S. \& Bothale K. (2008) Villousadenoma of the appendix with dysplasia. Saudi J Gastroenterol. 14.

Kehagias I, Zygomalas A, Markopoulos G, Papandreou T. \& Kraniotis P. (2016) Diagnosis and Treatment of Mucinous Appendiceal Neoplasm Presented as Acute Appendicitis. Case Rep Oncol Med. 2161952.

Kleiman DA, Finnerty B, Beninato T, Zarnegar R, Nandakumar G, Fahey TJ. \& Lee SW. (2015). Features Associated With Metastases Among Well-Differentiated Neuroendocrine (Carcinoid) Tumors of the Appendix:The Significance of Small Vessel Invasion in Addition to Size. Dis Colon Rectum. 58, 1137-43.

Madrigal S. \& Herrera G. (2018). Cáncer de apéndice: Revisión bibliográfica. Revista Clínica de la Escuela de Medicina UCR-HSJD 8.

Martínez J, Fumero L, Martínez V. \& Izquierdo E. (2018). Tumor carcinoide del apéndice cecal en mujer adolescente: a propósito de un caso. Rev Finlay 8.

Murphy E, Farguharson S. \& Moran B. (2006). Management of anunexpectedappendicealneoplasm. Br J Surg. 93,783-92.

Nutu O, Marcacuzco QA, Manrique MA, Alonso I, Calvo PJ, García CM, Cambra MF. \& Jiménez RL. (2017). Tumores mucinosos del apéndice: incidencia, diagnóstico y tratamiento quirúrgico. Rev Cirugía Española 95, 30558.

Pai RK. \& Longacre TA. (2005). Appendiceal mucinous tumors and pseudomyxoma peritonei: histologic features, diagnostic problems, and proposed classification. Adv Anat Pathol. 12, 291-311. 
Piamo et al.

Panomreongsak P, Sthapanachai C, Sukpanichnant S, Thakerngpol K, Sattawatthamrong Y, Wongsurakiat P, Wongwanit C. \& Seesillapachai P. (2000). Malignant carcinoid tumor of the appendix with liver and lung metastasis: report of a case with a high level of serum carcinoembryonic antigen. J Med Assoc Thai. 83, 97-102.

Rault-Petit B, Do Cao C, Guyétant S, Guimbaud R, Rohmer V, Julié C, Baudin E, Goichot B, Coriat R, Tabarin A, Ramos J, Goudet P, Hervieu V, Scoazec JY. \& WalterT. (2019). Current Management and Predictive Factors of Lymph Node Metastasis of Appendix Neuroendocrine Tumors: A National Study from the French Group of Endocrine Tumors (GTE). Ann Surg. 270, 165-71.

Rodríguez J, Delgado B, Dos Santos T, Llaque G. \& Moreno E. (2015). Tumores neuroendocrinos del apéndice cecal. Rev Venez. Oncol. 27, 171-7.

Rouchaud A, Glas L, Gayet M. \& Bellin MF. (2014). Appendiceal mucinous cystadenoma. Diagn Interv Imaging Internet. 95, 113-6.
Shaib WL, Goodman M, Chen Z, Kim S, Brutcher E, Bekaii-Saab T. \& El-Rayes BF. (2017). Incidence and survival of appendiceal mucinous neoplasms: a seer analysis. Am J Clin Oncol. 40, 569-73.

Sugarbaker P. (2006). The natural history, grosspathology and histopathology of appendiceal epithelial neoplasms. EJSO 32, 644-7.

Swank H, Eshuis E, Ubbink D. \& Bemelman W. (2010). Is routine histopathological examination of appendectomy specimen susefull a systematic review of the literature. Colorectal Dis. 13.

Wagner PL, Austin F, Sathaiah M, Magge D, Maduekwe U, Ramalingam L, Jones HL, Holtzman MP, Ahrendt SA, Zureikat AH, Pingpank J, Zeh HJ, Bartlett DL. \& Choudry HA. (2013). Significance of Serum Tumor Marker Levels in Peritoneal Carcinomatosis of Appendiceal Origin. Ann Surg Oncol Internet. 20, 50614. 